\title{
Marginal Mediation Analysis: A Practical Statistical Framework for Interpretable Mediation Effects
}

\author{
Tyson S. Barrett ${ }^{1}$, Ginger Lockhart ${ }^{1}$, \& Rick A. Cruz ${ }^{1}$ \\ ${ }^{1}$ Department of Psychology, Utah State University
}

\begin{abstract}
Mediation analysis is a widely used technique within the psychological sciences and has been shown to be an effective tool to evaluate explanatory pathways between predictors and outcomes. Multiple effect size metrics have been developed; however, mediation analysis has been slow to develop accessible, interpretable effect size metrics in the cases of categorical (or otherwise non-normally distributed) mediators and/or outcomes. Herein, we propose the use of average marginal effects within mediation analysis to alleviate these issues - termed Marginal Mediation Analysis. The method provides interpretable indirect and direct effect size estimates in the same units as the outcome even when mediators and/or outcomes are categorical, a count measure, or another non-normal distribution. The approach is shown to fit the causal definitions of mediation analysis. We further present results of Monte Carlo simulations that show the utility of the proposed method in psychological research. We also discuss the assumptions inherent in the approach. We conclude by showing an application of it to adolescent healthrisk behavior data $(\mathrm{n}=13,600)$, demonstrating the increased interpretability and information provided compared to other common approaches.
\end{abstract}

Keywords: mediation analysis, average marginal effects, regression analysis Word count: 6534

Investigators are often interested in not only if an effect exists from one variable to an outcome, but how that effect is transmitted through one or more intermediate variables. Mediation analysis is a widely used technique (MacKinnon, Fairchild, \& Fritz, 2007) that allows researchers to investigate this exact question, by providing a framework to evaluate the processes or pathways of an effect (Fairchild \& MacKinnon, 2009; Hayes, 2009; Iacobucci, 2008; MacKinnon, 2008; MacKinnon et al., 2007; Shrout \& Bolger, 2002). Generally speak-

Correspondence concerning this article should be addressed to Tyson S. Barrett, 2800 Old Main Hill, Logan, UT 84322. E-mail: tyson.barrett@usu.edu 


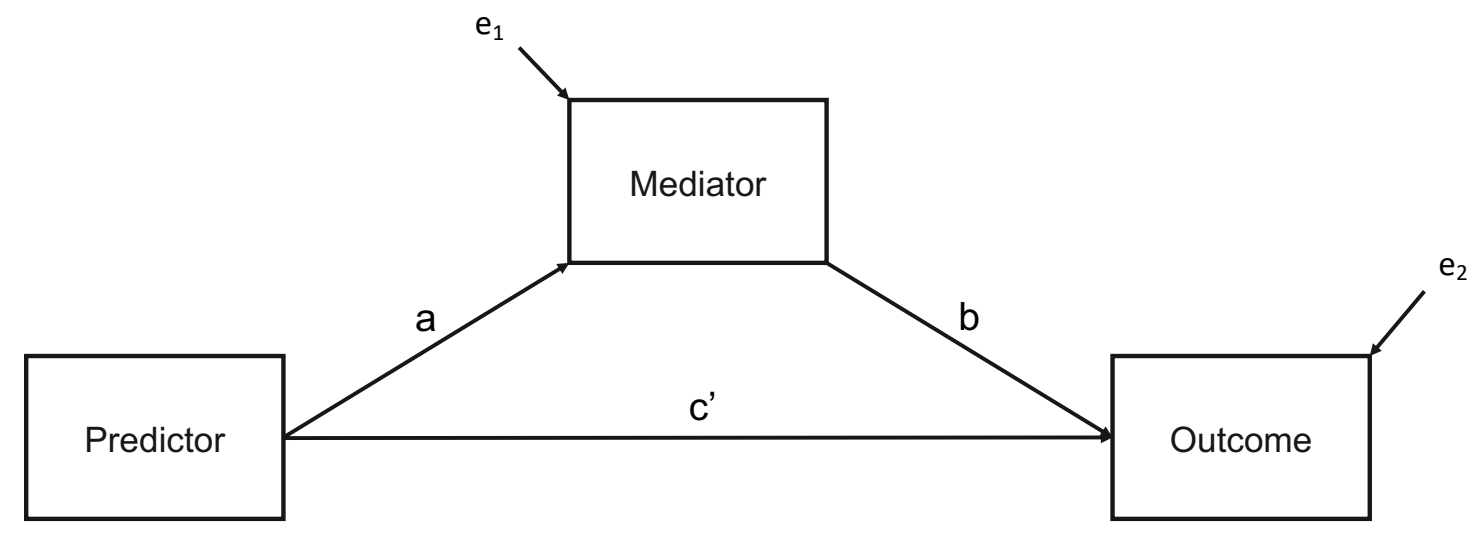

Figure 1. A diagram of a basic mediation model with the labels (e.g., a) being the common names of the pathways and $e_{1}$ and $e_{2}$ are the errors of the individual models.

ing, this is done by assessing how a given predictor influences a mediator which, in turn, affects an outcome (Serang, Jacobucci, Brimhall, \& Grimm, 2017). This is statistically made possible by combining two or more regression models to estimate each pathway of effect (Edwards \& Lambert, 2007; Hayes, 2009, 2013). To conceptualize and communicate these complex models, mediation models are often portrayed via path diagrams and directed acyclic graphs, as shown in Figure 1. Traditional confirmatory analysis within mediation is well established for a variety of situations (e.g., Lockhart, MacKinnon, \& Ohlrich, 2011) while exploratory analysis is beginning to take shape (Serang et al., 2017).

A benefit of mediation is that the concept is intuitive and results are generally interpretable. This, however, is generally only the case when mediators and outcomes are continuous. Once the data deviate from this, much of the intuition and interpretability can be lost. Significant advances have occurred regarding mediation analysis in the last decade especially in its connection to causal (counterfactual) effects. Interestingly, mediation with categorical or non-normal mediators/outcomes became more clear with these advancements (Pearl, 2012). However, the consistent application and interpretation is still somewhat elusive. That is, depending on the variable types of the mediator(s) and outcome(s), interpretation can rely on using specific equations to obtain the indirect and direct effects. For example, Muthén (2011) demonstrated the use of the causal framework to data that fit probit-type regression models. In order to obtain the causal effects, the use of probit-specific equations are needed. Although clearly possible to do, this creates more room for error in an already complex modeling approach.

Herein, we discuss a practical statistical framework for mediation analysis that has a consistent application and interpretation of the indirect and direct effects. This is made possible by using average marginal effects (AMEs) within mediation analysis. We demonstrate how average marginal effects provides a straightforward statistical approach in what is termed Marginal Mediation Analysis. First, we show how this approach relates to the causal mediation framework (Pearl, 2012). Next, we present how this new approach performs across different conditions via a series of Monte Carlo simulations. Third, we demon- 
strate how this novel method can improve the interpretability of mediation analysis with categorical and count data by comparing results from Marginal Mediation Analysis to results from traditional mediation analysis as shown in a prior study by Ford and Hill (2012) on the mediated role of religiosity on the risk of substance abuse.

\section{Current Use of Mediation Analysis}

With the recent advancements of mediation analysis, it is important to assess the use of mediation analysis in psychology and related fields. Studies were sought that saliently reported results wherein both mediation analysis and generalized linear models (e.g., logistic, probit) were used. Since $2013^{1}$, this produced 90 articles (via Scopus). ${ }^{2}$ Only articles that had a categorical/non-normal mediator and/or outcome was included in the review. Among these, three general categories of articles were found:

1. Articles that were methodologically building on mediation analysis (14\%). These included methodological papers, tutorials, and software papers.

2. Articles that applied mediation and the authors used the "difference method" (MacKinnon, 2008), or a close relative to the difference method (e.g., PROCESS), to assess the amount of mediation $(66 \%)$.

3. Articles that applied mediation and the authors used one of the various causal mediation approaches (often with structural equation modeling [SEM]) (20\%).

Although not a comprehensive review, this still highlights that the most popular approach wherein the mediation actually included a non-normal or categorical mediator/outcome relies on the difference method or a similar method.

The difference method relies on the following equations:

$$
\begin{aligned}
& a \times b+c^{\prime}=c \\
& a \times b=c-c^{\prime}
\end{aligned}
$$

where $a$ refers to the a path (the effect of the predictor on the mediator; see Figure 1), $b$ is the $b$ path (the effect of the mediator on the outcome), $c^{\prime}$ is the effect of the predictor on the outcome controlling for the mediator, and $c$ being the effect of the predictor on the outcome without controlling for the mediator.

In essence, this says that it is possible to estimate the indirect effect, that is $a \times b$ by assessing the difference $c-c^{\prime}$. However, it has some notable shortcomings. First, the standard errors can be inefficient and biased if there is a high degree of multi-collinearity (or the degree to which there is perfect separability) in any of the models. Second, it does not provide the effect size measures that would be most useful [e.g., the effect a change in the predictor has on the outcome through the mediator(s)]. Third, the difference method is consistently too conservative with binary outcomes (Jiang \& Vanderweele, 2015).

\footnotetext{
${ }^{1}$ The search interval from 2013 to 2019 was chosen to get a representative perspective on mediation analysis after the publication of causal mediation approaches.

${ }^{2}$ The search terms included: "mediation analysis" and ["logistic" or "probit" or "generalized linear models" or "GLM" or "Poisson"].
} 
In contrast, the currently less popular, but powerful, causal mediation approaches often use $\mathrm{SEM}^{3}$ with a counterfactual definition of the indirect and direct effects (Muthén \& Asparouhov, 2015; Muthén, 2011). The use of the counterfactual definitions is likely the best option currently available as it can provide results in intuitive metrics for indirect effects, direct effects, among others. However, its use requires an understanding of the counterfactual framework, including the equations to define each effect for each type of model. Although certainly possible to work with, this is not entirely practical, especially for less statistically-inclined researchers.

Ultimately, the recent use of mediation analysis highlights at least two notable patterns. First, the difference method may be popular, over that of the more informative causal approach, because it is the most intuitive approach to apply and does not require a background in understanding the counterfactual framework. Second, it is clear that mediation analysis with categorical or non-normal mediator(s) and/or outcome(s) is relatively common. Since these were the ones found where the researcher was attempting to adjust for the non-normal distributions, there are likely additional articles that failed to adjust for them. Together, these patterns suggest that a practical approach that allows interpretable mediated effect size estimates could improve the information gleaned mediation analyses in the field.

\section{Marginal Mediation Analysis}

Herein, we propose an accessible approach that provides interpretable effect sizes in mediation analysis in a way that applies across data situations (e.g., binary mediators, nominal outcomes, beta-distributed outcomes, etc.). It is made possible by integrating a post-estimation method known as average marginal effects within mediation analysis. This allows similar interpretations of all the paths and the indirect and direct effects in a mediation model with any type of mediator and/or outcome and can be shown to produce the causally-defined ${ }^{4}$ indirect effects and direct effects.

\footnotetext{
${ }^{3}$ Polychoric correlations for ordered categorical variables Muthén (1984) are also often used with categorical mediators and/or outcomes. Polychoric correlations are beneficial for ordinal data but otherwise are not designed to handle other types of categorical or non-normal data and lack the specific causal definition that the counterfactual framework affords.

${ }^{4}$ It is important to note that Marginal Mediation Analysis does not impact the causality of the overall conceptual model. Rather, it provides more flexibility in defining the model. To discuss causality in mediation analysis, one should be familiar with the ${ }^{*}$ causal* or *counter-factual* framework (or sometimes referred to as the potential outcomes framework; Hofler et al., 2005). As Imai et al. (2010a) states: "the causal effect ... can be defined as the difference between two potential outcomes: one that would be realized [in the intervention] and the other that would be realized if [not in the intervention]," (pg. 3). In other words, the causal effect is the difference in potential outcomes depending on the predictor (e.g., an intervention). In reality, only one such outcome is observed - if individual " $\mathrm{i}$ " is assigned to the treatment group, we only observe the outcome from the treatment group and not from the control group. Imai et al. (2010a) continues: "Given this setup, the causal effect of the [intervention] can be defined as $Y_{i}(1)-Y_{i}(0)$. Because only either $Y_{i}(1)$ or $Y_{i}(0)$ is observable ... researchers often focus on the identification and estimation of the average causal effect." If the conditions are randomly assigned, this is simply $E\left(Y_{i}(1)-Y_{i}(0)\right)$, or the expected value across multiple individuals and/or observations. The counter-factual framework helps clarify causality in mediation analysis by defining the necessary conditions. Using this framework, Imai et al. (2010b) demonstrated that *sequential ignorability* (essentially the assumption that there are no omitted influences) is required for a causal interpretation in mediation analysis. However, this assumption is difficult to assess. Because of this
} 
Beneficially, though, the use of average marginal effects produces the indirect and direct effects without having to adjust the approach for differing variable types. Rather, the research models each path with the appropriate link and distribution (e.g., logistic regression uses a binomial distribution with a logit link, linear regression uses a normal distribution with an identity link) and provides this model to the general mediation model. The resulting effects will be calculated automatically using average marginal effects and does not require any model-specific definition of the mediated paths.

To understand how this synthesis of mediation analysis and AMEs helps solve the main issues resulting from categorical and non-normal mediators/outcomes, we first review average marginal effects.

\section{Average Marginal Effects}

Average marginal effects allow generalized linear models (GLMs) to have interpretations similar to that of linear regression. This is beneficial because linear regression, unlike GLMs, is interpreted in terms of the marginal effects - the effect a small change in the predictor has on the outcome in the units of the outcome. To have this type of interpretation, AMEs use a post-estimation calculation. That is, the generalized linear model is unchanged until after the estimates have been made. Once the model has been fit to the data and the estimates are calculated, AMEs take the estimates and the original data to find the marginal effect for each predictor for each observation in the model. The average across observations of the marginal effects for each variable is the average marginal effect for that variable.

To help illustrate this, consider the example of a logistic regression. To obtain the marginal effect of say, $X_{1}$, we need to find the partial derivative with respect to $X_{1}$ (i.e., the marginal effect of $X_{1}$ ) as shown in the equations below.

$$
\begin{gathered}
\operatorname{logit}\left(Y_{i}\right)=\beta_{0}+\sum_{j=1}^{p} \beta_{j} X_{i j}+\epsilon_{i} \\
\text { marginal effect } X_{X_{1}}=\frac{\delta Y}{\delta X_{1}}=\frac{e^{\beta_{0}+\sum_{j=1}^{p} \beta_{j} X_{i j}+\epsilon_{i}}}{\left(1+e^{\beta_{0}+\sum_{j=1}^{p} \beta_{j} X_{i j}+\epsilon_{i}}\right)^{2}}
\end{gathered}
$$

This means that the marginal effect of the predictor $X_{1}$ depends on the level of each covariate for each individual (all $X_{i j}$ 's) and each estimate (all $\beta_{j}$ 's). Thus, each observation and each variable in the model has its own marginal effect; some individuals will have larger marginal effects of a variable while others have smaller effects regarding that variable. By taking the average of these marginal effects, we obtain the AME for that variable. In other words, the AME is the associated average change in the outcome for a one unit increase in $X_{1}$.

In an instructive paper about the (now defunct) routine called "margeff" in Stata, Bartus (2005) highlights how the AME can be calculated-including the mathematical

difficulty, Imai et al. (2010a) developed a general mediation model that allows a researcher to assess how deviations from it, via sensitivity analysis, affect the estimates. 
definition - and the benefits of AMEs compared to other related methods. For a continuous variable, the AME is defined as:

$$
A M E_{k}=\beta_{k} \frac{1}{n} \sum_{i=1}^{n} f(\beta X)
$$

where $f$ refers to the probability density function of $F, \beta X$ is the linear combination of the predictors (i.e., the model predicted values for each observation), and $A M E_{k}$ is the average marginal effect for the $k t h$ variable. This definition provides the average change in the outcome for a one unit change in the continuous variable $x_{k}$ across all $n$ observations.

Relatedly, the AME of a dummy coded variable is:

$$
A M E_{k}=\frac{1}{n} \sum_{i=1}^{n}\left[F\left(\beta X \mid x_{k i}=1\right)-F\left(\beta X \mid x_{k i}=0\right)\right]
$$

where $F\left(\beta X \mid x_{k i}=1\right)$ is the predicted value of the $i t h$ observation when the dummy variable $x_{k}$ equals one and $F\left(\beta X \mid x_{k i}=0\right)$ is the predicted value when the dummy value of $x_{k}$ equals zero holding all other variables constant. This, in effect, shows the average discrete difference between the levels of the categorical variable in the outcome's original units.

Since AMEs are estimates, it is important to understand the uncertainty behind the estimates. Two general approaches are used. The first approach is the delta method, which provides standard errors (StataCorp, 2015). Although beneficial, the second-bootstrapped confidence intervals - have been accurate for understanding the variability in both AMEs and mediation analysis. We, therefore, use bootstrapping herein.

\section{The Proposed Marginal Mediation Analysis}

Building on the mediation framework discussed by Hayes (2009) and by Edwards and Lambert (2007), MMA is established on linear regression maximum likelihood. In this framework, two or more regression equations are combined to provide the overall mediation model. This method then adds a post-estimation step into this mediation framework.

The form of the general marginal mediation model are demonstrated in the following equations, where Equations 3 and 4 demonstrate the mediation estimation while Equations 5 and 9 show the post-estimation procedures.

$$
\begin{gathered}
M_{i j}=a_{0}+\sum_{k=1}^{p} a_{k} x_{k i}+\epsilon_{1 i} \quad \text { for } \mathrm{j}=1, \ldots, \mathrm{m} \text { mediators, and } \\
Y_{i}=\beta_{0}+\sum_{j=1}^{m} b_{j} M_{i j}+\sum_{k=1}^{p} c_{k}^{\prime} x_{k i}+\epsilon_{2 i}
\end{gathered}
$$

for the $i t h$ individual, for $k=1, \ldots, p$ predictors, and $j=1, \ldots, m$ mediators. The paths are all labeled with their common term (e.g., path $a$ is labeled $a$ ). Combining these two equations provides the full mediation model. Using these models, we apply the post-estimation 
of the average marginal effects as presented by Bartus (2005). For a continuous $x_{k}$ variable, the average marginal effect of path $a$ is:

$$
A M E_{k}^{a}=a_{k} \frac{1}{n} \sum_{i=1}^{n} f(a X)
$$

where $f$ refers to the probability density function, $a X$ is the linear combination of the predictors (i.e., the model predicted values for each observation), and $A M E_{k}^{a}$ is the average marginal effect of the $a$ path for the $k t h$ variable. Ultimately, Equation 5 is identical to that of the following:

$$
A M E_{k}^{a}=\frac{1}{n} \sum_{i=1}^{n} \frac{F\left(a X_{1}\right)-F\left(a X_{2}\right)}{2 h}
$$

where

$$
a X_{1}=\left[\begin{array}{cccccc}
a x_{11} & a x_{12} & \ldots & a x_{1 k}+h & \ldots & a x_{1 p} \\
a x_{21} & a x_{22} & \ldots & a x_{2 k}+h & \ldots & a x_{2 p} \\
\vdots & \vdots & \ddots & \vdots & \ddots & \vdots \\
a x_{n 1} & a x_{n 2} & \ldots & a x_{n k}+h & \ldots & a x_{n p}
\end{array}\right]
$$

and

$$
a X_{2}=\left[\begin{array}{cccccc}
a x_{11} & a x_{12} & \ldots & a x_{1 k}-h & \ldots & a x_{1 p} \\
a x_{21} & a x_{22} & \ldots & a x_{2 k}-h & \ldots & a x_{2 p} \\
\vdots & \vdots & \ddots & \vdots & \ddots & \vdots \\
a x_{n 1} & a x_{n 2} & \ldots & a x_{n k}-h & \ldots & a x_{n p}
\end{array}\right]
$$

Both $F\left(a X_{1}\right)$ and $F\left(a X_{2}\right)$ are the model predicted value for the outcome given a small change denoted $h$. Equations 6, 7, and 8 use a very small $h$ value (default is $1 \times 10^{-7}$ ). This provides the change in the average predicted value for a very small increase and a very small decrease in in the $x_{k}$ variable. This is also described in depth by Leeper (2017) as it is the strategy employed by the margins $\mathrm{R}$ package.

Similarly, the AME of a dummy coded variable in the $a$ path is:

$$
A M E_{k}^{a}=\frac{1}{n} \sum_{i=1}^{n}\left[F\left(a X \mid x_{k i}=1\right)-F\left(a X \mid x_{k i}=0\right)\right]
$$

where $F\left(a X \mid x_{k i}=1\right)$ is the predicted value of the $i t h$ observation when the dummy variable equals one and $F\left(a X \mid x_{k i}=0\right)$ is the predicted value when the dummy value equals zero. Notably, these same post-estimation equations ( 5 and 9) can be used for the $b$ and $c^{\prime}$ paths as well.

Assumptions of Marginal Mediation Analysis. The statistical estimation of MMA is based on a number of assumptions (as discussed by MacKinnon (2008)).

1. No Omitted Influences. A key to any mediation analysis is that variables that: 1) correlate with both the predictor and the mediator (path $a$ ), 2) correlate with both the mediator and the outcome (path $b$ ), or 3 ) correlate with both the predictor and the outcome (path $c^{\prime}$ ) are included in the model. A more general form of this assumption has been termed "sequential ignorability" (Imai et al., 2010a). This more general form includes a sensitivity analysis to assess how deviations from this assumption can affect the conclusions (Imai et al., 2010a, 2010b). 
2. Accurate Measurement. Random measurement error produces attenuated paths (in large sample sizes) and random bias (in small sample sizes) in regression (Loken \& Gelman, 2017) and therefore can affect the paths in various ways (e.g., attenuate the $b$ path which can inflate the $c^{\prime}$ path). When possible, reliable measures and/or proper latent variable modeling should be used for this assumption to be met.

3. Well-Behaved Residuals. The residuals are assumed to be random, "have constant variance at each value of the predictor variable" and "residual error terms are uncorrelated across equations" (pg. 55). This assumption can be related to "No Omitted Influences" for, if there are omitted variables in both equations, the error terms will correlate.

4. No Predictor-Mediator Interaction. In most cases, we assume that the predictor and the mediator do not interact in their effect on the outcome. This assumption can be relaxed but must be specified within the model. This interaction changes the conceptual model and, thus, the interpretation of the results.

5. Correct Functional Form. The proper form of the model needs to be specified, including the distribution and link of the modelling procedure. In general, mediation also assumes a linear relationship between predictors and mediators/outcomes. This can relaxed by using GLMs to transform the outcome to provide a linear relationship.

\section{Comparison to the "Causal Mediation Model" from Pearl (2012) and Múthen (2011)}

Given the causal (counterfactual) mediation approach makes the indirect, direct, and total effects clear, it is important to compare MMA to the causal definitions clarified by Pearl (2012) and explicated in regards to SEM by Muthén (2011). Below, we compared the two approaches analytically, showing the similarity between the approaches.

First, assume that we have the following mediation model, built of the following regression equations:

$$
\begin{gathered}
y_{i}=\beta_{0}+\beta_{1} m_{i}+\beta_{2} x_{i}+\epsilon_{1 i} \\
m_{i}=\gamma_{0}+\gamma_{1} x_{i}+\epsilon_{2 i}
\end{gathered}
$$

where $y_{i}$ is the outcome with an arbitrary distribution and $m_{i}$ is the mediator with an arbitrary distribution. That is, we assume a mediation model with a single predictor, mediator, and outcome (for simplicity, although the results extend naturally to more predictors and mediators).

The indirect effect from MMA results in:

$$
\begin{gathered}
I E_{A M E}=A M E_{a} \times A M E_{b} \\
I E_{A M E}=\frac{1}{n} \sum_{i=1}^{n}\left[F_{1}\left(\gamma_{1} x \mid x=1\right)-F_{1}\left(\gamma_{1} x \mid x=0\right)\right] \\
\times \frac{1}{n} \sum_{i=1}^{n}\left[F_{2}\left(\beta_{1} m+\beta_{2} x \mid m=1\right)-F_{2}\left(\beta_{1} m+\beta_{2} x \mid m=0\right)\right] .
\end{gathered}
$$


This can be rewritten in general form as

$$
I E_{A M E}=E\left[M\left(x^{\prime}\right)-M(x)\right] \times E\left[Y\left(m^{\prime}, x\right)-Y(m, x)\right]
$$

where $E\left[M\left(x^{\prime}\right)-M(x)\right]$ is the expected change in $M$ for a small change in $x$ and $E\left[Y\left(m^{\prime}, x\right)-Y(m, x)\right]$ is the expected change in $Y$ for a small change in $m$ holding $x$ constant at $x$. Thus, the first part is the amount that $M$ changes for a change in $x$ to $x^{\prime}$ while the second part is the change in $Y$ for a change in $m$ to $m^{\prime}$. In other words, the equation is weighted to only change $Y$ by the amount that would occur with a change in $M$ of the size that would occur if $x$ was changed to $x^{\prime}$.

This result can be compared to the definition provided by Pearl (2012). As Pearl states: "The indirect effect... is defined as the expected change in $Y$ affected by holding $X$ constant, at $X=x$, and changing $[\mathrm{M}]$ (for each individual) to whatever value it would have attained had $X$ been set to $X=x^{\prime}$," (pg. 7; known as the "Mediation Formula"). The equation can be written as:

$$
I E_{\text {Causal }}=\sum_{z} E(Y \mid x, M)\left[P\left(M \mid x^{\prime}\right)-P(M \mid x)\right]
$$

where $E(Y \mid x, M)$ is the expected change in $Y$ for a change in $M$, holding $X=x$, while $P\left(M \mid x^{\prime}\right)-P(M \mid x)$ is the change in the probability of $M$ given a change in $X$ from $x$ to $x^{\prime}$. This can be rewritten for a our mediation model as

$$
I E_{\text {Causal }}=E\left[Y\left(m^{\prime}, x\right)-Y(m, x)\right] \times E\left[M\left(x^{\prime}\right)-M(x)\right] .
$$

Thus, both the causal framework and MMA produce the indirect effect as the expected change in $Y$ when the mediator is changed by what is expected by a change from $x^{\prime}$ to $x$ while controlling for $X$. That is, the effect of $X$ on $Y$ through $M$.

As for the direct effect, the definition with AME is:

$$
D E_{A M E}=A M E_{c}=\frac{1}{n} \sum_{i=1}^{n}\left[F_{2}\left(\beta_{1} m_{i}+\beta_{2} x_{i} \mid x_{i}=1\right)-F_{2}\left(\beta_{1} m_{i}+\beta_{2} x_{i} \mid x_{i}=0\right)\right]
$$

which can be written as:

$$
D E_{A M E}=E\left(Y \mid m, x^{\prime}\right)-E(Y \mid m, x) .
$$

Interestingly, this is identical to the "controlled direct effect" discussed in Pearl (2012) and Múthen (2011):

$$
C D E_{\text {Causal }}=E\left(Y \mid m, x^{\prime}\right)-E(Y \mid m, x) .
$$

Pearl (2012) importantly states that the value at which $M$ is held constant is important in non-linear models. In the AME framework, this value will be held at the observed values of $M$ in the sample. Notably, Appendix I shows a replication of one of the analyses in Muthén (2011) showing MMA and causal mediation providing identical indirect effects. 


\section{Monte Carlo Simulations}

Given the assumptions of the model hold, we assessed the general performance of MMA via Monte Carlo simulations, in the $\mathrm{R}$ statistical environment version 3.5.1. The focus of the simulations was to gauge the 1) consistency regarding how closely $a \times b+c^{\prime}$ is to $c$ (i.e., does the indirect plus the direct effect equal the total effect?), 2) statistical power (i.e., how often does the null properly get rejected?), 3) bias (i.e., is the mean of the estimates at the population mean?), and 4) confidence interval coverage (i.e., does the confidence interval for the indirect effects contain the population value in the right proportion of models?).

Population Models. The data were simulated from a known population with a researcher specified causal model (i.e., the "population model"). The model consisted of either a binary mediator ( $0=$ "No", $1=$ "Yes") or a count mediator (Poisson distribution), a continuous outcome, and a continuous predictor. The sample size and the effect sizes were also varied for a total of 90 unique combinations of the conditions (see Table 1). The effect sizes for both the binary mediator and count mediator (a path) were the odds ratios and risk ratios corresponding to small, moderate, and large effect sizes. These are found in Table 2. Here, 500 data sets were simulated for each combination of experimental conditions (Carsey \& Harden, 2013; Paxton, Curran, Bollen, Kirby, \& Chen, 2001).

The $a$ path population model is defined below for when the mediator is binary, where the $\operatorname{Prob}\left(M_{u}=1\right)$ is a latent continuous variable with a logistic relationship with the predictors and $\epsilon_{i}$ is normally distributed with a mean of 0 and a standard deviation of 1 .

$$
\log \left(\frac{\operatorname{Prob}\left(M_{u}=1\right)_{i}}{1-\operatorname{Prob}\left(M_{u}=1\right)_{i}}\right)=a_{0}+a_{1} x+\epsilon_{i}
$$

The observed variable, $M_{o}$, is defined as:

$$
M_{o}= \begin{cases}0, & \text { if } \operatorname{Prob}\left(M_{u}=1\right)<.5 \\ 1, & \text { otherwise }\end{cases}
$$

The $a$ path population model for when the mediator is a count is shown below where $M_{u}$ is a latent continuous variable with an exponential relationship with the predictors and the $\epsilon_{i}$ is normally distributed with a mean of 0 and a standard deviation of 1 .

$$
\log \left(M_{u}\right)=a_{0}+a_{1} x+\epsilon_{i}
$$

The observed variable, $M_{o}$, is defined as follows:

$$
M_{o}=\operatorname{Po}\left(\lambda=M_{u}\right)
$$

This creates an observed, count variable that has $\lambda$ values based on the latent mediator.

The $b$ and $c^{\prime}$ paths population model is identical to Equation 4 with only one predictor and a single binary or count mediator, with a continuous outcome, as shown below.

$$
Y_{i}=\beta_{0}+b M_{i}+c^{\prime} x_{i}+\epsilon_{y i}
$$

Table 1 highlights the conditions that were varied for each simulation. A distinct MMA model was applied to each of the 500 data sets for each possible combination of experimental conditions. In total, 45,000 MMA models were assessed. 
Table 1

The various experimental conditions of the Monte Carlo simulation study.

\begin{tabular}{lc}
\hline Independent Variables & Conditions \\
\hline Sample size & $50,100,200,500,1000$ \\
Effect size of a path & small, moderate, large \\
Effect size of b path & small, moderate, large \\
Effect size of c' path & moderate \\
Type of mediator & binary, count \\
\hline Total conditions & 90 \\
\hline
\end{tabular}

Table 2

The effect sizes of the $a$ and $b$ paths in the Monte Carlo Simulation.

\begin{tabular}{lccc}
\hline Size & Odds Ratio (a path) & Risk Ratio (a path) & r (b path) \\
\hline Small & 1.58 & 1.34 & 0.10 \\
Moderate & 3.44 & 1.82 & 0.30 \\
Large & 6.73 & 3.01 & 0.50 \\
\hline
\end{tabular}

Decomposed Total Effect Equals The Total Effect. One of the major questions about the performance of MMA regards whether the decomposed total effect equals the total effect $\left(a \times b+c^{\prime}=c\right)$. Table 3 highlights the average discrepancy between the decomposed total effect and the total effect divided by the total effect (thereby adjusting the discrepancy for the size of the total effect). On average, for each sample size tested deviations are small, generally $<.5 \%$ of a discrepancy, with the majority $<.1 \%$. The average discrepancies also decrease in size as the sample size increases.

Table 3

Average discrepancies between the decomposed total effect and the total effect for various sample sizes.

\begin{tabular}{lcc}
\hline & \multicolumn{2}{c}{ Mediator } \\
Sample Size & Binary & Count \\
\hline 50 & 0.0017 & -0.0080 \\
100 & 0.0024 & -0.0028 \\
200 & $<0.001$ & 0.0027 \\
500 & $<0.001$ & -0.0036 \\
1000 & $<0.001$ & -0.0027 \\
\hline
\end{tabular}

Figure 2 presents the individual simulated differences between the decomposed total effect and the total effect. Once assessing the individual discrepancies, two patterns are of note: 1) there are larger discrepancies for smaller sample sizes and larger effect sizes; and 2 ) besides a single outlier in the count condition (Panel b), most discrepancies are small.

First, the largest discrepancies are where the sample sizes are small $(\mathrm{n}=50)$ and the effect sizes are larger. For both binary and count mediators, the discrepancies, even 
in the large effect sizes, are very small as the sample increases to $\mathrm{n}=1000$. Second, in the count condition there is a clear outlying value $(>2$ for the $\mathrm{n}=50$ and large effect size condition). Other than this value - across the binary and count mediators - all other values are relatively close to zero. For the binary mediator condition, the scale was in risk (probability) units. The discrepancies in the $\mathrm{n}=50$ condition are notable in their size while the other conditions had discrepancies that are essentially within rounding error. For the count mediator condition, the scale of the total effect was in count units. The outlier is notable in its large discrepancy in these units; most other values were essentially within rounding error of the effect size.

Statistical Power. Figure 3 shows the average statistical power of MMA across the various conditions (i.e., the statistical power at each tested sample size for each combination of effect sizes). Most effect size combinations are adequately powered at a sample size of 200 across both binary and count mediator conditions. Interestingly, the small by small condition had more power at higher sample sizes than large by small, which is contrary to intuition. However, the issue here was the issue of complete separability wherein the estimates and the standard errors are either biased or not estimable in logistic regression. With a large effect and a small sample size, this became common, thus reducing the statistical power in these conditions. The count mediator condition did not have this issue.

Bias. It is also important for MMA to estimate the expected parameters. Figure 4 highlights that MMA is consistent in estimating the underlying effects for each combination of effect sizes across the various sample sizes. In the figure, which is stratified by the combination of effect sizes, shows the population parameter (vertical line) and the estimated values (the density distributions). Overall, the distributions are centered at the true population parameter in each situation across the conditions.

Confidence Interval Coverage. The confidence interval coverage is shown in Figure 5. Although the coverage is approximately $95 \%$, it is clear that there is some deviation from the $95 \%$ line, particularly in the binary mediator condition. This is not a major deviation but an important one, nonetheless.

\section{Discussion of Monte Carlo Simulations}

Although just a first look at the behavior of MMA, the results indicated it is a viable approach to working with both binary mediators and count mediators. It provides an approach that allows the $a \times b+c^{\prime}=c$ condition to approximately hold. It has the statistical power for even very small indirect effects with a sample size of 1000 . Furthermore, across all tested conditions, the average of the estimates accurately identified the population parameter.

However, the approach can be improved in various ways, including the confidence interval coverage. Given the use of the percentile bootstrapping method herein, it may be important to apply other bootstrapping approaches such as the Bias-Corrected Bootstrap. This finding of the indirect effect having confidence intervals that were too narrow has been found previously for the percentile bootstrapped (as applied in MMA; MacKinnon, Lockwood, \& Williams, 2004). However, MacKinnon et al. (2004) also found the bootstrap methods, including the percentile approach, is among the best of the tested approaches. Other approaches, including the Monte Carlo confidence interval could be integrated to 
provide an empirical $p$-value in future studies.

\section{Application of MMA}

In 2012, Ford and Hill published a study that used some of the most common approaches to mediation when a mediator and/or outcome is categorical. Specifically, they used:

1. the difference method (MacKinnon, 2008),

2. the "categorical data method outlined by MacKinnon (2008)" (pg. 5) to assess the significance of the difference method, and

3. the percent of the total effect that was mediated.

These three approaches are common, as shown earlier. However, these only provide part of the information about mediation and are often biased with categorical mediator(s) and outcome(s).

To build on the important findings from Ford and Hill (2012), this study replicates their work using more recent data from 2014. In addition, we add the use of MMA to obtain effect sizes and confidence intervals for the indirect and direct effects.

The descriptive statistics are found in Table 4 for the 13,600 adolescents in the sample. Overall, these sample statistics were very similar to the 2007 sample used by Ford and Hill. However, the prevalence of drug use across each category dropped since 2007, although marijuana use did not drop substantially (13.85\% in 2007 to $12.7 \%$ in 2014). Heavy drinking (10.4\% in 2007) had only a single positive response in the entire sample of adolescents in 2014. The number of major depressive episodes increased from $8.4 \%$ in 2007 to $11.3 \%$ in 2014. Attitudes regarding drug use were essentially identical as that in 2007 for the respondent, peer, and parent (and each had high reliabilities - all $\alpha \geq .80$ - comparable to 2007). The attitudinal measures and the measure of religiosity had high reliabilities as well.

First, we replicated the main methods employed by Ford and Hill, including the significance test for the difference method of the indirect effects and discussed the percent of mediation. The percent of mediation reported was based on the $c-c^{\prime}$ from the logistic regression models predicting each outcome. Each model controlled for parental conservative attitudes toward substance use, the adolescents' family income, and the adolescents' age, race, and sex. Table 5 presents the amount of the total effect of religiosity on substance use that is mediated through respondent conservative attitudes, peer conservative attitudes, and depression. ${ }^{5}$ Overall, the effect of religiosity on substance use is heavily mediated by the hypothesized mediators, more so for tobacco use than the others. The significance test, as done by Ford and Hill, is highlighted in the table, showing each indirect effect was statistically significant.

Next, we used four MMA models to assess the pathways from adolescent religiosity to substance use, one for each outcome (any tobacco use, prescription drug misuse, marijuana

\footnotetext{
${ }^{5}$ Because MMA provides information about each of the indirect effects naturally in the same units, it is possible to assess the amount mediated by each mediator while also controlling for the other mediators in a straightforward manner-without having to fit several other models and assess each $c-c^{\prime}$.
} 
Table 4

Descriptive statistics of the sample.

\begin{tabular}{lc}
\hline & $\begin{array}{c}\text { Percent } / \text { Mean (alpha) } \\
\mathrm{n}=13,600\end{array}$ \\
\hline Drug use & $5.7 \%$ \\
Prescription drug misuse (past year) & $11.8 \%$ \\
Tobacco use (past year) & $0 \%$ \\
Heavy drinking (past 30 days) & $12.7 \%$ \\
Marijuana use (past year) & $3.5 \%$ \\
Other illicit drug use (past year) & \\
Demographics & $49.0 \%$ \\
Female & $45.8 \%$ \\
Race (Non-White) & $55.4 \%$ \\
Income (2x poverty level) & $11.3 \%$ \\
Major Depression Episode & \\
Attitudinal measures & $2.6(\alpha=.86)$ \\
Respondent (range 1-3) & $2.5(\alpha=.88)$ \\
Peer (range 1-3) & $2.9(\alpha=.84)$ \\
Parent (range 1-3) & $\alpha=.80$ \\
Religiosity &
\end{tabular}

use, and other illicit drug use). As before, each model controlled for parental conservative attitudes toward substance use, the adolescents' family income, and the adolescents' age, race, and sex for each path. Figure 6 presents the individual paths in the model units. Therefore, the paths leading to the conservative attitudes (both respondent and peer attitudes) are in the attitude metric with a range from 1 - 3. The paths leading to depression and the substance outcomes are all in log-odds. As the figure highlights, most paths were statistically significant at $\mathrm{p}<.05$.

In addition to these results, MMA provides information regarding the indirect and direct effects in the same units. Figure 7 highlights the indirect and direct effects with their associated $95 \%$ confidence intervals. All of the effects here are in change of risk (probability) units - i.e., the change in the risk of tobacco use, prescription misuse, marijuana use, or

Table 5

The percent of mediation (the percent of the total effect) by path for each outcome.

\begin{tabular}{lcccc}
\hline \multirow{2}{*}{ Mediator } & \multicolumn{4}{c}{ Outcome } \\
\hline Respondent Views & $34.2^{*}$ & $14.0^{*}$ & $23.2^{* * *}$ & $14.1^{* *}$ \\
Peer Views & $23.2^{*}$ & $22.0^{*}$ & $15.8^{* * *}$ & $17.7^{* *}$ \\
Depression & $4.0^{*}$ & $9.2^{*}$ & $1.8^{* * *}$ & $4.4^{* *}$ \\
Total Mediation & 61.4 & 45.2 & 40.8 & 36.1 \\
\hline
\end{tabular}

Note: ${ }^{* * *} \mathrm{p}<.001,{ }^{* *} \mathrm{p}<.01,{ }^{*} \mathrm{p}<.05$ 
illicit drug use for a one unit change in religiosity. Although all indirect effects and most direct effects are significant, the effect size estimates are particularly important here as the meaningfulness of these significant effects can be overlooked. On their own, these effects appear small.

We can also look at how big the effect is compared to the overall proportion of risk for that outcome. For this situation the average risks are $11.8 \%$ for tobacco use, $5.7 \%$ for prescription drug misuse, $12.7 \%$ for marijuana use, and $3.5 \%$ for illicit drug use. For tobacco, then, the average risk is $11.8 \%$ while the total effect of religiosity is 2.1 percentage points. This results in about an $18 \%$ decrease in risk from the average $((11.8-2.1) / 11.8)$ for a one unit increase in religiosity. For prescription drug misuse the effect is about $24.5 \%$ decrease in risk from the average; for marijuana use the effect is about a $31 \%$ decrease in risk from the average; and for illicit drug use, the effect is approximately a $34 \%$ decrease in risk from the average, all for a one unit increase in religiosity.

Together, these findings highlight three main ideas. First, the effect of religiosity on substance use is heavily mediated through conservative respondent views toward substance use, conservative peer views toward substance use, and depression. This means, if assumptions hold, religiosity exerts much of its impact on drug use by influencing views on substance use, by providing opportunities to have peers that have conservative views on substance use, and to a lesser extent, by reducing depression. Second, in absolute terms, the effect of religiosity on substance use is small, only moving the risk by a few percentage points. Third, relative to the average risks of using the various substances, the effect is moderate, showing changes ranging from 18 to $31 \%$ decreases in risk from the average.

\section{Summary of Marginal Mediation Analysis}

The results across the Monte Carlo simulations and the application of MMA demonstrate the utility of the new approach in mediation-based research. First, the approach matches the well-defined causal mediated effects. Second, the simulation results highlight its ability to estimate the effects consistently, with statistical power to detect even small effect sizes with a sample size of 1000. Third, the application highlights that the effect sizes produced by MMA, especially the indirect and direct effect sizes, are valuable companions to the information generally provided by mediation with categorical/non-normal mediators and/or outcomes. These effect sizes can highlight the importance of any significant effects and provide context to the meaningfulness of the effects beyond that of simply discussing the percent mediated.

Importantly, if the outcome is continuous, MMA can produce standardized effect sizes for each path and the indirect, direct, and total effects. This is done by normalizing the outcome variable. For categorical predictors, this is sufficient to obtain a standardized mean difference effect size for each path and effect. For continuous predictors, one must normalize the continuous predictors which will provide a standardized effect size that is interpreted based on the standard deviations of the variables. For categorical outcomes, MMA will not standardize given the results are already in units that are somewhat "standardized" (e.g., probabilities, counts).

The application of MMA is notable in that it is not more complex than using the 
difference method with its estimate of significance, or other related approaches (e.g., PROCESS). As in the others, we need to run regression models for each path. But then MMA combines them into a single conceptual model with the indirect and direct effects estimated. In terms of complexity, the actual performance of the modelling is straightforward while the theoretical designing of the conceptual model is, and should be, far more involved.

Finally, MMA provides several avenues for interpretation, allowing the researchers to highlight important findings and transparently show trends and patterns across the model. The approach provides information on the individual paths along with the individual model fits, information on the standardized and unstandardized indirect and direct effects, and in some situations, the effects can be compared to baseline rates to obtain context for the size of the effect.

Limitations. Some limitations should be acknowledged regarding MMA and the approaches used to assess it. First, the implementation currently only handles a single set of predictors, a single set of mediators, and an outcome. That is, the model cannot assess predictor to mediator to another mediator to outcome. Second, the implementation is currently only in R, although other platforms are planned. Third, MMA is only as good as the theoretical model behind it. If the model specifications are wrong, MMA cannot solve those problems. Fourth, the Monte Carlo simulations were built to meet the assumptions of the modeling and therefore may not apply when assumptions are violated.

Future Directions. Several avenues exist to further develop and test MMA. The approach needs to be tested in other modeling situations, including with other mediator/outcome distributions, with interactions, with missing values, in multilevel data, and in complex-sampling siutations. It would also be important to compare the utility and behavior of an MMA-like approach in situations with latent variables. Because MMA is built on maximum likelihood regression as is most latent variable modeling approaches, the integration should be possible. Finally, it is important to assess the performance of MMA in situations where assumptions are violated or otherwise unlikely to hold. For these situations, it would be important to integrate innovative approaches, such as the sensitivity analysis (Imai et al., 2010a), used to assess the impact violations of specific assumptions.

\section{Conclusion}

Marginal Mediation Analysis is an approach that makes mediation analysis, regardless of mediator and outcome distributions, interpretable. It has been shown herein to fit several of the definitions of causal mediated effects. It was also shown to be consistent at estimating the population values and is statistically well-powered when assumptions hold. The use of MMA also highlighted some important effect sizes in the mediated role of religiosity on reducing substance use. Ultimately, this practical statistical framework can help researchers apply mediation in more complex data situations.

\section{Open Science Statement}

The data and code to run all analyses for the application can be found at osf.io/an7hg. Furthermore, an R package with the functionality to run MMA (MarginalMediation) is available for free (Barrett, 2019). 


\section{References}

Barrett, T. S. (2019). MarginalMediation: Marginal mediation. Retrieved from https:// CRAN.R-project.org/package=MarginalMediation

Bartus, T. (2005). Estimation of marginal effects using margeff. Stata Journal, 5(3), 309-329. doi:The Stata Journal

Carsey, T. M., \& Harden, J. J. (2013). Monte carlo simulation and resampling methods for social science. SAGE.

Edwards, J. R., \& Lambert, L. S. (2007). Methods for integrating moderation and mediation: a general analytical framework using moderated path analysis. Psychological Methods, 12(1), 1-22. doi:10.1037/1082-989X.12.1.1

Fairchild, A. J., \& MacKinnon, D. P. (2009). A general model for testing mediation and moderation effects. Prevention Science, 10(2), 87-99. doi:10.1007/s11121-008-0109-6

Ford, J. A., \& Hill, T. D. (2012). Religiosity and Adolescent Substance Use: Evidence From the National Survey on Drug Use and Health. Substance Use 85 Misuse, 47(7), 787-798. doi:10.3109/10826084.2012.667489

Hayes, A. F. (2009). Beyond Baron and Kenny: Statistical Mediation Analysis in the New Millennium. Statistical Mediation Analysis in the New Millennium, 76(4), 408-420. doi:10.1080/03637750903310360

Hayes, A. F. (2013). Introduction to mediation, moderation, and conditional process analysis: A regression-based approach. The Guilford Press.

Hofler, M. (2005). Causal inference based on counterfactuals. BMC Medical Research Methodology, 5(28), 1-12. doi:10.1186/1471-2288-5-28

Iacobucci, D. (2008). Mediation analysis. SAGE [Kindle Version].

Iacobucci, D., Saldanha, N., \& Deng, X. (2007). A mediation on mediation: Evidence that structural equation models perform better than regression. Journal of Consumer Psychological, 7(2), 140-154. doi:10.1016/S1057-7408(07)70020-7

Imai, K., Keele, L., \& Tingley, D. (2010a). A general approach to causal mediation analysis. Psychological Methods, 15(4), 309-34. doi:10.1037/a0020761

Imai, K., Keele, L., \& Yamamoto, T. (2010b). Identification, Inference and Sensitivity Analysis for Causal Mediation Effects. Statistical Science, 25(1), 51-71. doi:10.1214/10STS321

Jiang, Z., \& Vanderweele, T. (2015). When is the difference method conservative for assessing mediation? American Journal of Epidemiology, 182(2), 105-108. doi:10.1093/aje/kwv059

Leeper, T. J. (2017). Interpreting Regression Results using Average Marginal Effects with $R$ 's margins (pp. 1-31). Retrieved from https://cran.r-project.org/web/packages/ margins/vignettes/TechnicalDetails.pdf 
Lockhart, G., MacKinnon, D. P., \& Ohlrich, V. (2011). Mediation analysis in psychosomatic medicine research. Psychosomatic Medicine, 73(1), 29-43. doi:10.1097/PSY.0b013e318200a54b.Mediation

Loken, E., \& Gelman, A. (2017). Measurement error and the replication crisis. Science, 355(6325), 584-585. doi:10.1126/science.aal3618

MacKinnon, D. P. (2008). Introduction to statistical mediation analysis. Routledge.

MacKinnon, D. P., Fairchild, A. J., \& Fritz, M. (2007). Mediation Analysis. Annual Review of Psychology, 58, 593-602. doi:10.1146/annurev.psych.58.110405.085542.Mediation

MacKinnon, D. P., Lockwood, C. M., \& Williams, J. (2004). Confidence Limits for the Indirect Effect: Distribution of the Product and Resampling Methods. Multivariate Behavioral Research, 39(1). doi:10.1207/s15327906mbr3901_4

Muthén, B., \& Asparouhov, T. (2015). Causal effects in mediation modeling: An introduction with applications to latent variables. Structural Equation Modeling: A Multidisciplinary Journal, 22(1), 12-23.

Muthén, B. O. (2011). Applications of Causally Defined Direct and Indirect effects in mediation analysis using SEM in Mplus.

Paxton, P., Curran, P. J., Bollen, K. a., Kirby, J., \& Chen, F. (2001). Monte Carlo Experiments: Design and Implementation. Structural Equation Modeling: A Multidisciplinary Journal, 8(2), 287-312. doi:10.1207/S15328007SEM0802_7

Pearl, J. (2012). The Causal Mediation Formula-A Guide to the Assessment of Pathways and Mechanisms. Prevention Science, 13(4), 426-436. doi:10.1007/s11121-011-0270-1

Serang, S., Jacobucci, R., Brimhall, K. C., \& Grimm, K. J. (2017). Exploratory Mediation Analysis via Regularization. Structural Equation Modeling: A Multidisciplinary Journal, 1-12. doi:10.1080/10705511.2017.1311775

Shrout, P. E., \& Bolger, N. (2002). Mediation in experimental and nonexperimental studies: new procedures and recommendations. Psychological Methods, 7(4), 422. doi:10.1037//1082-989x.7.4.422

StataCorp. (2015). Stata Statistical Software: Release 14. StataCorp LLC. 


\section{Appendix I}

In addition, we replicated the results shown in Table 7 of Múthen (2011), given the data were available and the analysis was relevant to MMA. Results were, as expected, nearly identical in regards to the indirect effect size estimate (-.022 and -.021 for Múthen and MMA, respectively) while there was more of a discrepancy with the direct effect size estimate (-.050 and -.043 for Múthen and MMA, respectively). The difference in the direct effects is likely because the MMA approach uses the controlled direct effect while Múthen (2011) used the natural direct effect. Importantly, all conclusions were identical in terms of significance and directionality of the effect. 

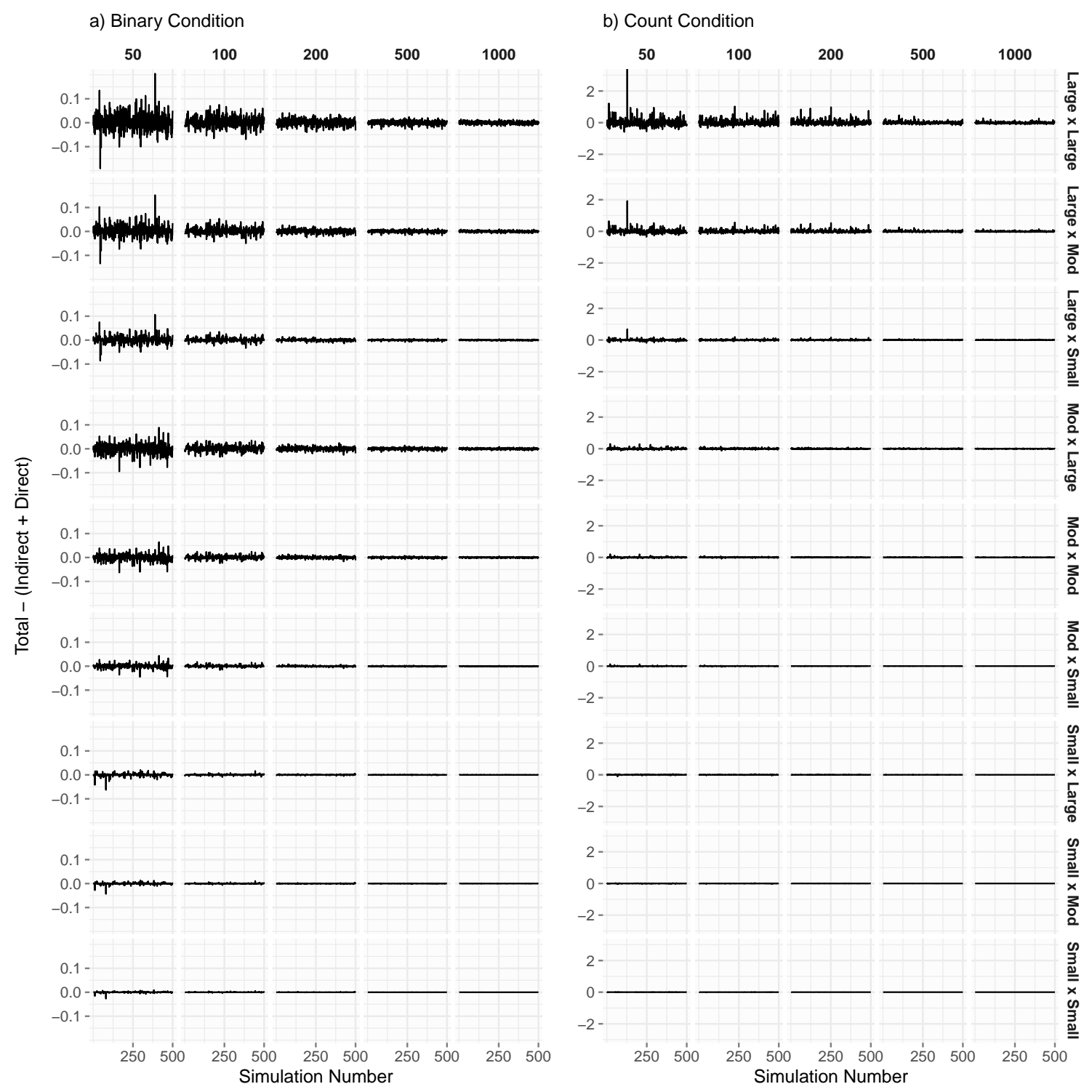

Figure 2. The simulated differences between the decomposed total effect and the total effect. The discrepancies are higher for smaller sample sizes and larger effect sizes. 


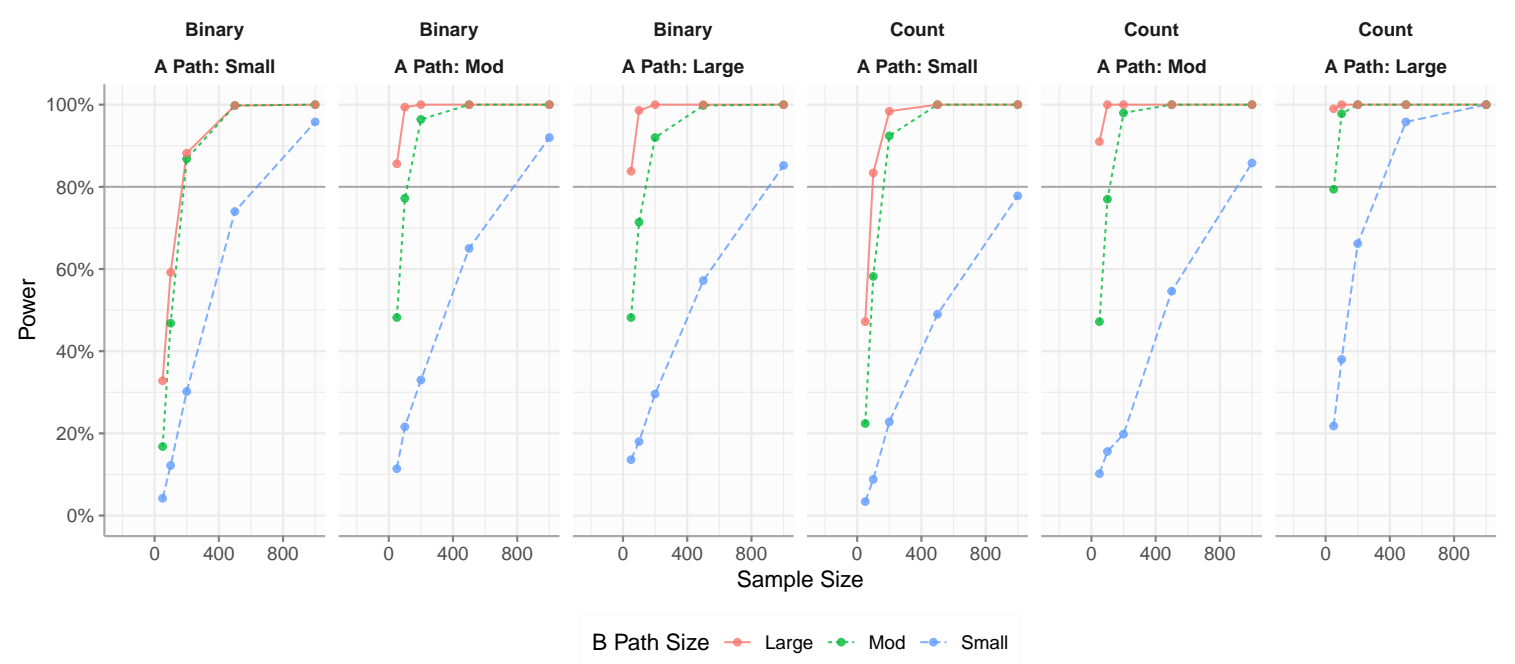

Figure 3. The simulated levels of power per tested sample size (x-axis) and effect size of the $\mathrm{b}$ path, stratified by the distribution of the mediator (binary or count) and the size of the a path.
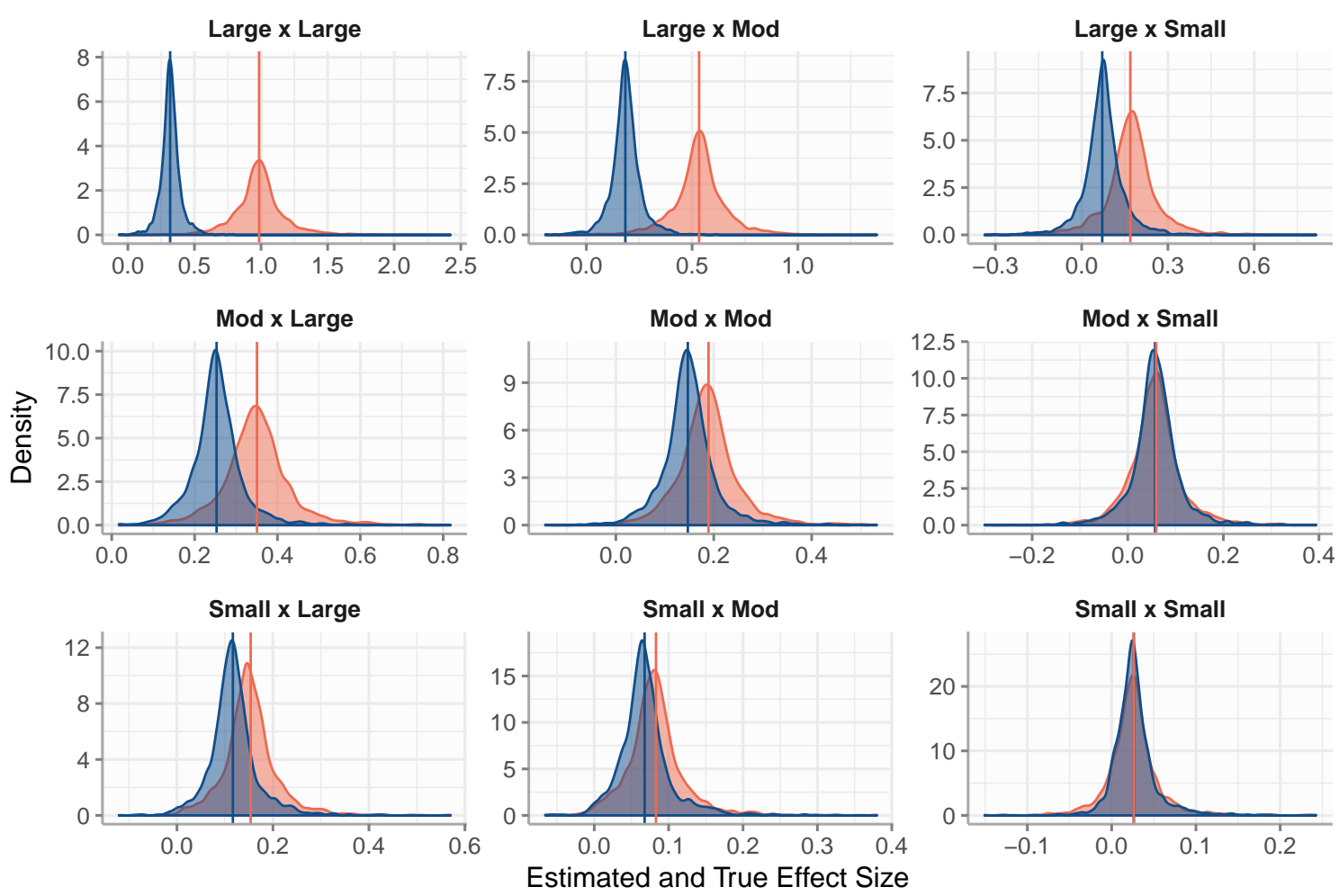

Mediator Distribution $\square$ Binary $\square$ Count

Figure 4. The simulated accuracy per tested sample size (x-axis) and effect size of the indirect path (color; a combination of the a path by the b path). 


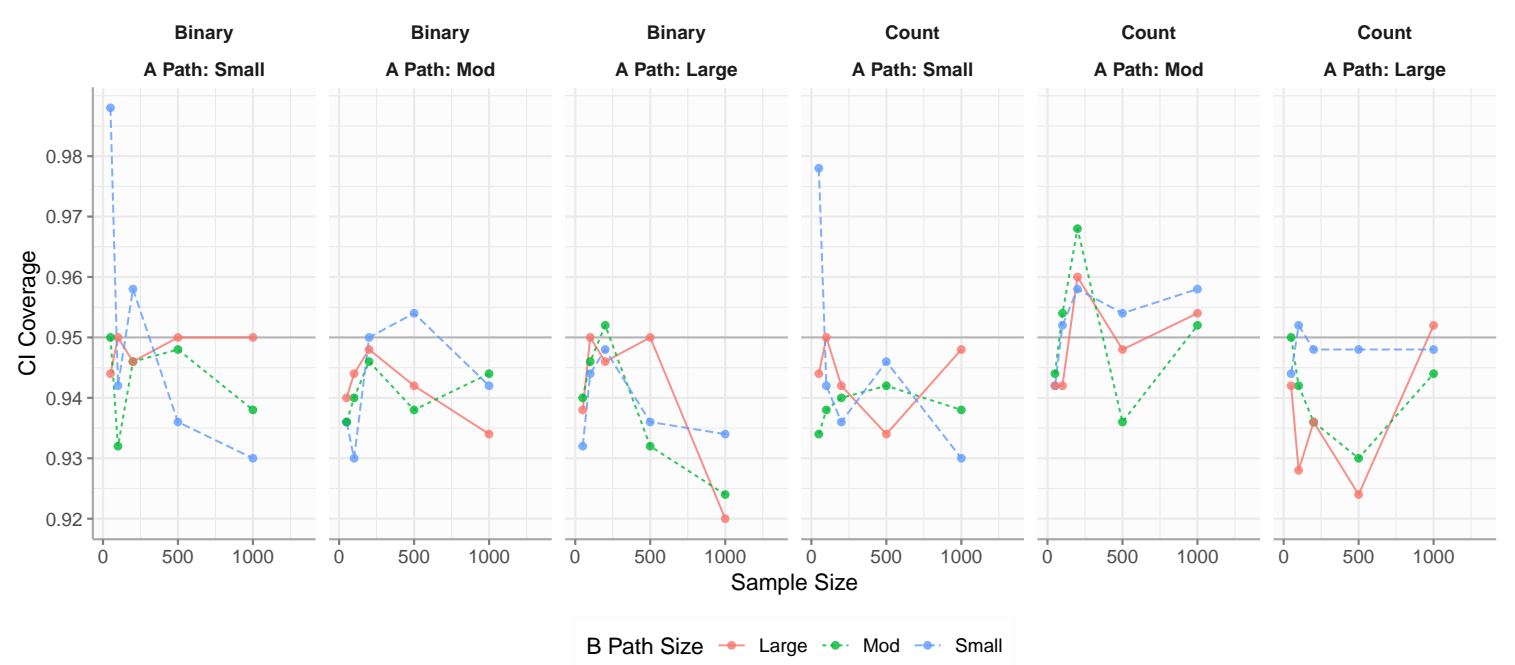

Figure 5. The simulated confidence interval coverage per tested sample size (x-axis) and effect size of the b path, stratified by the distribution of the mediator (binary or count) and the size of the a path.

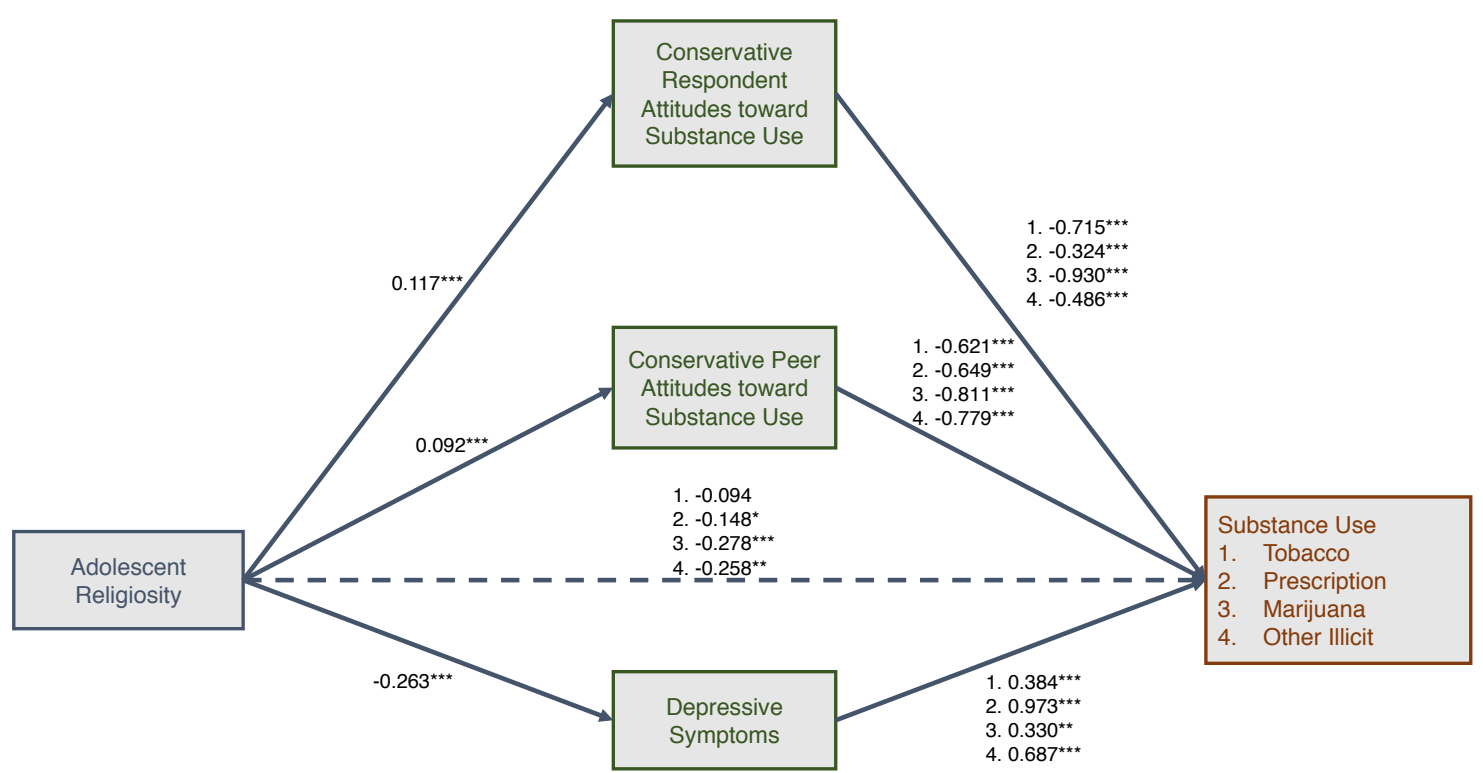

Figure 6. Results of the mediation models' individual paths regarding religiosity and substance use. Note: ${ }^{* * *} \mathrm{p}<.001,{ }^{* *} \mathrm{p}<.01,{ }^{*} \mathrm{p}<.05$ 

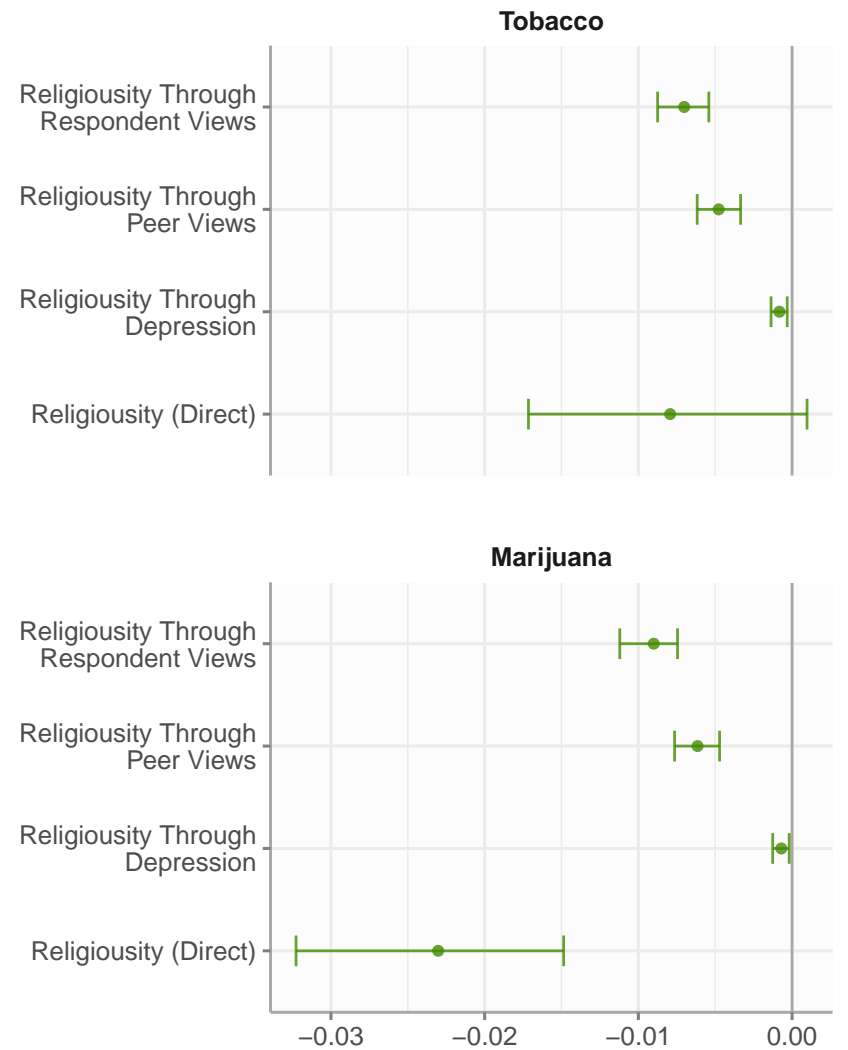

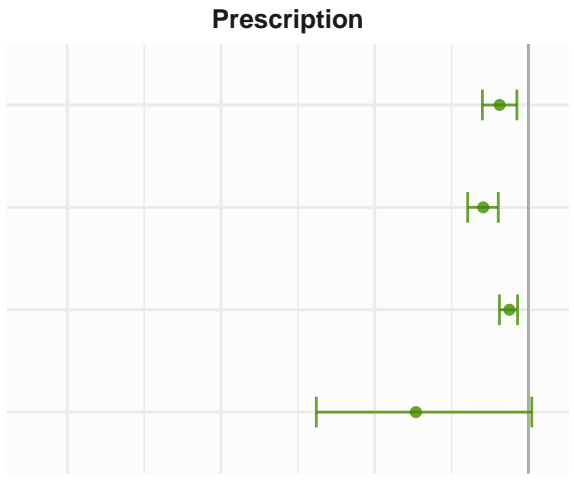

Illicit

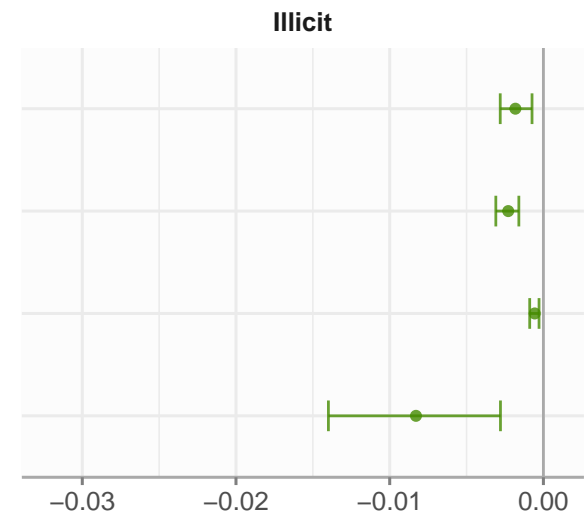

Figure 7. Comparison of the effect sizes of the indirect and direct effects across the four outcomes. 